\title{
Special Transmission Gear Invalidation Analysis Coupled with Finite El- ement Method Based on Meshless Local Petrov-Galerkin Method
}

\author{
Guangbin $\mathrm{Yu}^{1,2}$, Xuemei $\mathrm{Wu}^{1 *}$, Di Liu ${ }^{2}$, Chang-sheng $\mathrm{Hu}^{1}$, Fan Zhang ${ }^{3}$,Bing Dai ${ }^{2}$ \\ and Debin Shan ${ }^{1}$ \\ ${ }^{1}$ Harbin Institute of Technology, Harbin, 150001, China \\ ${ }^{2}$ Harbin University of Science and Technology, Harbin, 150080, China \\ ${ }^{3}$ The Seventh Aerospace Research Institute, The Seventh Department of Integrative \\ System Design, Chengdu, Sichuan Provincial 610100
}

\begin{abstract}
Aiming at high nonlinear problem of special transmission gear invalidation analysis, provided a method which based on Meshless local Petrov-Galerkin method coupled with finite element method and method to solve fracture problem of special transmission gear. Simulation calculation has been done to non-involute beveloid gear developed by project team. Calculation result verified efficiency of the simulation method. The method has important meaning to novel gear development and research.
\end{abstract}

Keywords: Finite element method; Meshless local Petrov-Galerkin method; Special transmission gear; Invalidation analysis

\section{Introduction}

The rapid development of modern machinery industry led the development of new types of gears, while the design and development process requires a simulation tool to verify the correctness of the design to reduce the development cycle and funding. Bending fracture of the tooth is one of the most common failure mode, for the purpose of early detection and prevention, the calculation should be taken in the early period of the design.

Gear failure often occurs because of the stress concentration at the tooth surface and tooth root locally. It is a high nonlinear problem, so it is difficult to make accurate dynamic simulation using finite element method. Element-free method is another widely used structural analysis method following the finite element method. As a numerical solution of partial differential equations, it has an advantage in computational accuracy over the local stress concentration, and it can handle highly nonlinear local issues, such as large deformation and fracture issues etc. it is a good supplement of finite element method in solving discontinuous problems. Therefore, a calculation method of dynamic simulation is promoted for the gear failure on the basis of MLPG [1-2] (Meshless local PetrovGalerkin).

\section{MLPG and the Improvement}

MLPG method is a mature and mesh free computing approach. In comparison with the finite element method, It is free from the binding of mesh, Trial function based on the nodes which discretized the solution domain. So it could solve the high non-linear and discontinuous problem. 


\subsection{Interpolation}

Moving least squares method is used to fit the trial function, and the PU method is one of the least squares methods with simple form which is widely used. The prototype of PU functions (partition functions) is given by [3].

$$
\varphi_{i}(x)=W_{i} / \sum_{j=1}^{N} W_{j}
$$

Where $\varphi_{i}(x)$ is shape functions of node $i ; \mathrm{N}$ is the number of nodes within the definition of interpolation points; where $\mathrm{W}$ is the weight function of node ${ }^{i}$.

The difficulty of numerical integration should be considered while choosing the weight functions. In order to facilitate the Gaussian integral, quartic spline curve is selected as the weight function [4].

$$
\omega_{i}(s)=\left\{\begin{array}{lc}
1-6 s^{2}+8 s^{3}-3 s^{4} & 0 \leq s<1 \\
0 & s \geq 1
\end{array}\right.
$$

Where $s=\left(\left\|x-x_{i}\right\|\right) / r$, here $\left\|x-x_{i}\right\|$ is the distance between nodes I and the interpolation point, and $\mathrm{R}$ is the radius of influence domain.

\subsection{Coupling Algorithm}

In the moving least square method, shape function is formulated by the weight function of the node within the domain. By changing some of the weight functions of the nodes, the improved shape function of this node is much larger than the other nodes to make the trial function to wear through the node.

Where function $\alpha$ is composed of the Exponential Functions which have the compact support property and spike characteristic, and peak characteristics can be adjusted through the coefficient $\beta$.

$$
\omega_{\text {inew }}(x)=\left(\delta \cdot \alpha(x) \omega_{i}(x)\right)
$$

Function $\alpha$ is given by

$$
\alpha(x)=\left\{\begin{array}{c}
c e^{-\left(\frac{h}{\beta}\right)^{2}}, h \leq 1 \\
0, h>1
\end{array}\right.
$$

Where $h=\frac{d}{R_{i}}$, and $\mathrm{d}$ is the distance between interpolation point and the node $i ; R_{i}$ denotes the influence domain radius of node $i ; \beta$ is the coefficient of the function and $\beta \in[0,1]$. In this paper, $\beta$ is 0.4 ; $\mathrm{b}$ is the Dirac function when the node $\mathrm{i}$ is a border node $\delta=1$, otherwise $\delta=0$; c is the coefficient:

$$
c=c_{0} \sum_{i=1}^{n} \omega_{i}
$$

Where $n$ is the number of nodes within the definition domain; $\omega_{i}$ is weight function 
of node $i ; c_{0}$ is a constant, and $c_{0} \geq 15$.

After improving the power function, the node shape functions have approximate properties with delta function, and maintain the smooth characteristic of the original shape function. Figure 1 is the shape function curves of one-dimensional problem, and 50 nodes are equidistant arranged in the interval $[0,30]$, with the influence domain radius of 30. Figure 1 shows the drawing of a section of the node shape function, and the shape function value at nodes is approximately equal to 1 .

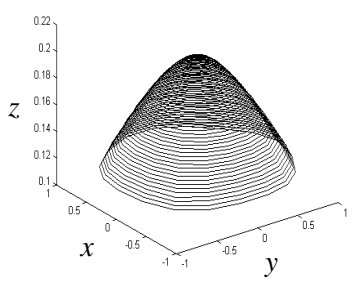

a) before improved

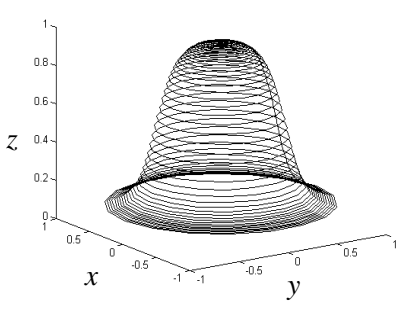

b)after improved

Figure.1. The Improved Shape Function

\subsection{Equivalent of the Weak Form}

The solution domain $\Omega$ is shown in Figure 2, and the control equations and boundary conditions are given by:

$$
\left\{\begin{array}{l}
\sigma_{i j, j}+\bar{f}_{i}=0 \\
\sigma_{i j} \cdot n_{j}-\bar{t}_{i}=0 \\
u_{i}-\bar{u}_{i}=0
\end{array}\right.
$$

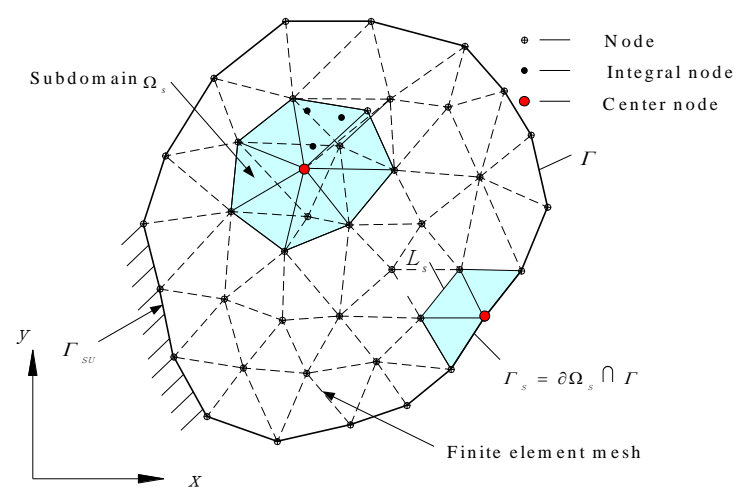

Figure 2. The Solution Domain and The boundary Diagram

Weak form of the equivalent integral is given by

$$
{ }_{\Omega}^{\prime}\left(\sigma_{i, j}+\bar{f}_{i}\right) N_{i} d \Omega-\alpha_{\Gamma_{s u}^{\prime}}(\bar{u}-u) N_{i} d \Gamma=0
$$

Where $\mathrm{N}$ is the test function (Test Function), and distribute the first term of equation (7). 


$$
{ }_{\Omega}^{\prime} \sigma_{i, j} N_{i} d \Omega={ }_{\Omega}\left(\sigma_{i j} N_{i}\right) \frac{\partial\left(\sigma_{i j} N_{i}\right)}{\partial u_{j}} j d \Omega-\sigma_{\Omega} \sigma_{i j} N_{i, j} d \Omega
$$

Using Gauss theorem, the first term on the right of equation (8) can be written as.

$$
{ }_{\Omega_{i}} \sigma_{i j} N_{i, j} d \Omega-{ }_{r_{m}^{\prime}} t_{i} N_{i} d \Gamma={ }_{r_{s}} \bar{t}_{i} N_{i} d \Gamma+{ }_{\Omega_{s}} \bar{f}_{i} N_{i} d \Omega
$$

The division of the boundary A shows as follows

$$
\sigma=D B \phi U
$$

Decompose the right term of equation (10)

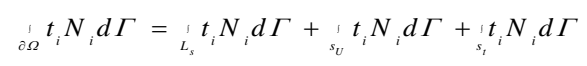

It is difficult to solve the first term of the equation on the right. However, by choosing the appropriate test function, we can make the value of the test function in the sub-domain boundary integral is zero, so that the first term of the equation on the right can be eliminated. In this paper, the triangular element shape function in the finite element method is used as the test function, and weight function is zero in the sub-domain boundaries which has compact support features. Where $N_{i}$ is the test function of the Integration point $i$, namely the finite element shape functions of the triangle area around the center node shows as follows.

$$
N_{i}=\frac{1}{2 A}\left(a_{i}+b_{i} x+c_{i} y\right)
$$

The test function value at the center node is 1 with the shape of tent, and at the boundary the value is 0 .

Where $\mathrm{A}$ is the triangle's area of the integral point, and a, b and c are the coefficient related with the node. from equation (5) (8) and equation (4) we can obtain

$$
\begin{aligned}
& { }_{\Omega}^{\prime} \sigma_{i j} N_{i, j} d \Omega+\alpha \underset{\Gamma_{u t}}{ } u_{i} N_{i} d \Gamma-\underset{\Gamma_{u i}}{t_{i}} N_{i} d \Gamma
\end{aligned}
$$

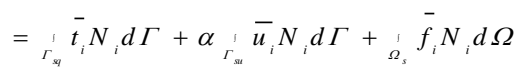

In this paper, an improved PU function is used as shape function, displacement boundary conditions are met in advance, so equation (13) can be written as

$$
{ }_{\Omega_{s}} \sigma_{i j} N_{i, j} d \Omega-{ }_{r_{\omega}} t_{i} N_{i} d \Gamma={ }_{r_{\omega}} \bar{t}_{i} N_{i} d \Gamma+{ }_{\Omega_{s}} \bar{f}_{i} N_{i} d \Omega
$$

Where $\sigma_{i j}$ is the stress matrix and it can be expressed as a function of the displacement. tensor form shows as follows .

$$
\sigma_{i}=D_{i j} B_{j k} \phi_{k m} U_{m}
$$

The equivalent matrix form is given by 
Where $\sigma$ is the stress vector, and $\sigma=\left[\sigma_{1}\right]$; D denotes constitutive matrix; $\mathrm{B}$ is the differential operator matrix; $\phi$ is the shape function matrix; $U$ denotes the displacement vector. Solving equation (15), the displacement field can be obtained, according to equation (15) or (16) stress field can be obtained.

\subsection{Discrete Form of the Control Equation}

The equation (14) can be written in matrix form.

$$
K \cdot U=F
$$

Where $\mathrm{U}$ is the displacement vector, and $U^{T}=\left[\bar{u}_{1}\right] ; F$ is the load vector, and $F=F_{1}+F_{2}$; $K$ is the stiffness matrix and $K=K_{1}+K_{2}$.

Stiffness matrix $K$ can be written as the sum of $K_{1}$ and $K_{2}$ matrix, where matrix, and $K_{1}$ is given by

$$
K_{1}=(J \cdot W) V^{T} D B \Phi
$$

Where $J$ are the vector of the Jacobi values of the different Gaussian integration points; $W$ are the vectors composed of the weights of different integration points; $D$ is derivative matrix of the test the function and can be written as

$$
V^{T}=\left[\begin{array}{ccc}
N, x & 0 & N, y \\
0 & N, y & N, x
\end{array}\right]
$$

$D$ is the constitutive matrix, and for linear elastic materials it can be written as

$$
D=D_{0}\left[\begin{array}{ccc}
1 & v_{0} & 0 \\
v_{0} & 1 & 0 \\
0 & 0 & \frac{1-v_{0}}{2}
\end{array}\right]
$$

$B$ is the differential operator matrix and can be written as

$$
B=\left\{\begin{array}{cc}
\partial / \partial x & 0 \\
0 & \partial / \partial y \\
\partial / \partial y & \partial / \partial x
\end{array}\right]
$$

$\Phi$ is the shape function matrix, and can be written as

$$
\Phi=\left[\begin{array}{ccccc}
\varphi_{1} & 0 & \varphi_{2} & 0 & \cdots \\
0 & \varphi_{1} & 0 & \varphi_{2} & \cdots
\end{array}\right]
$$


In the shape function, the presence of discontinuous or sharp point would led to numerical integration difficulties and low accuracy problem. The quartic spline curve is used as the weight function in this element-free method, and PU function as the shape function. its derivative is given by

$$
\frac{\partial F_{i}}{\partial x}=\frac{d w x}{W}+\frac{w_{i} \partial W}{W^{2} \partial x}
$$

Where ${ }_{W}=\sum_{i} w_{i}$, and $d w x$ is the derivative of weight function; in solving domain, the derivative of the weight function of node i shows as follows

$$
\begin{gathered}
\frac{\partial w_{i}}{\partial x}=\left[-12\left(\frac{d_{i}}{r}\right)+24\left(\frac{d_{i}}{r}\right)^{2}-12\left(\frac{d_{i}}{r}\right)^{3}\right] \frac{\partial\left(d_{i} / r\right)}{d x} \\
\frac{\partial d_{i}}{\partial x}=\frac{2\left(x-x_{0}\right)}{\sqrt{\left(x-x_{0}\right)^{2}+\left(y-y_{0}\right)^{2}}}=\frac{2\left(x-x_{0}\right)}{d_{i}}
\end{gathered}
$$

From equation (25) and equation (24) we can obtain equation (26)

$$
\frac{\partial w_{i}}{\partial x}=\left[-12\left(\frac{d_{i}}{r}\right)+24\left(\frac{d_{i}}{r}\right)^{2}-12\left(\frac{d_{i}}{r}\right)^{3}\right] \frac{2\left(x-x_{0}\right)}{r d_{i}}
$$

Matrix $K_{2}$ can be written as

$$
K_{2}=J W n N{ }^{T} D B F
$$

Where $\mathrm{n}$ is the test function of integration point, and $N$ is the matrix of the normal direction of the edge expressed as

$$
N^{T}=\left[\begin{array}{ccc}
n_{1} & 0 & n_{2} \\
0 & n_{2} & n_{1}
\end{array}\right]=\left[\begin{array}{ccc}
\sin \theta & 0 & \cos \theta \\
0 & -\cos \theta & \sin \theta
\end{array}\right]
$$

$\theta$ is the angle between the boundary normal and the horizontal axis.

\section{Discontinuities Algorithm}

As shown in Figure 3, before gear failure there are surface cracks. The influence domain of the node $x_{b}$ which is near to the cracks covers the cracks, so the shape function value $\phi_{b} \neq 0$ which is the following parts under the crack (shaded parts), namely the upper and lower parts of the cracks are continuous, which does not match the reality, so in this paper modified weight function is used to solve this problem. The nodes in the solving domain are divided into three parts: node $x_{b}$ near the crack border, node $x_{t}$ near the crack tip and the other nodes. Three kinds of nodes have different weight functions, the weight function of the nodes near the boundary crack on the other side is zero, that is to say, the nodes do not affect each other on both sides of the cracks, and each domain does 
not cover each other. The weight functions of the nodes near the crack tip are relatively complex. The ray model is used in the text, so a ray can be established through the node $y{ }^{c}$ with the origin of ${ }^{x}$. The solving domain is divided into two parts by this ray and cracks, and the value of the weight function of $x_{t}$ is zero in the other side of the solution domain $\left(V_{d}\right)$.

Fault line is described by a number of lines ordered counterclockwise, and suppose the length of the line is R. For any node ${ }^{i}$ located within solving domain, it should be determined whether it is on either the left, the right or the fracture line. As shown in Figure 4, the endpoint $y_{c i}$ which is the nearest fault line point from the node $i$ should be identified, and endpoints before and after endpoint $y_{c i}$ are described as ${ }_{c i-1}$ and $y_{c i+1}$ respectively; then, the end point will be selected between $y_{c i-1}$ and $y_{c i+1}$ which is nearer to the node i, so a triangle can be constituted by this point, the end point $y_{c i}$ and the node ${ }^{i}$, where $P_{1}$ is the node ${ }^{i}, P_{2}$ and ${ }^{P_{3}}$ represent the nearer end points of the two fault lines; Finally, the final judging results can be get by eqution (29), and the weight function can be improved by the function $g^{c(x)}$.

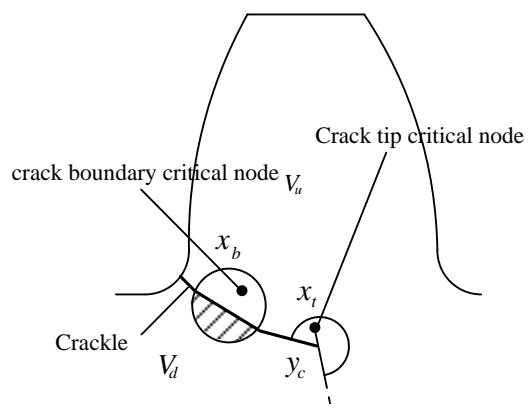

Figure 3. Treatment of Crack Border

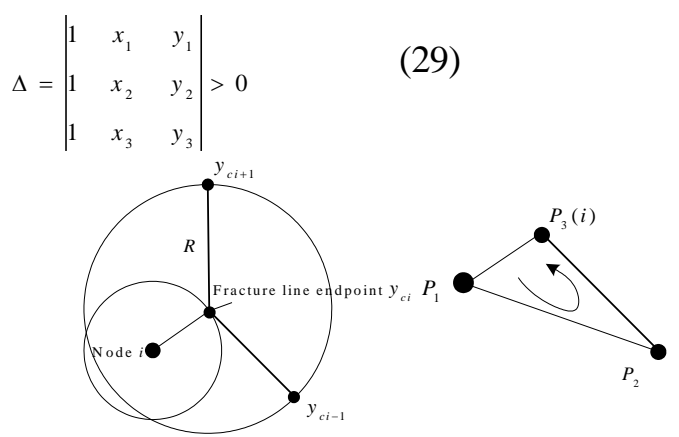

Figure 4. The Region Judgments of the Nodes

$$
\begin{cases}\Delta>0 & g c(x)=1\left(X \in V_{u}\right) \\ \Delta<0 & g c(x)=-1\left(X \in V_{p}\right) \\ \Delta=0 & g c(x)=0\end{cases}
$$

The amended weight function is given by : 


$$
W_{\text {new }}(x)=d(x) W_{\text {old }}(x)
$$

Where $d_{(x)}$ is the correction term.

$$
d(x)= \begin{cases}0 & g c(x) g c\left(x_{a}\right)=-1 \\ 1 & \text { other }\end{cases}
$$

Crack tip field function is singular with the feature $\sqrt{r}$, so the following basic function $p(x)$ is used:

$$
p(x)=\left\{1, x_{1}, x_{2}, \sqrt{r}\right\}
$$

\section{Strength Analysis of Cross-axis Thickening of Non-involute Gears}

\subsection{The Background}

At present, many imported machines apply cross-axis thickening gears. However, the existing types of gear transmission are the areas of space points meshing, so contact strength is low and carrying capacity is small. Cross-axis thickening of non-involute gear transmission is a new kinds of gear transmission [5]. As shown in Figure 5, this gear drive resolve the low strength problem of such gears by achieving a line contact. In order to verify the design of the cross-axis thickening of non-involute gear transmission, the strength analysis is explored.

Parameters of Gear 1: normal module ${ }^{m_{n 1}}=8 \mathrm{~mm}$, normal pressure angle ${ }^{\alpha_{n 1}}=20^{\circ}$, the number of teeth $z_{1}=29$, modification coefficient ${ }^{x_{n 1}}=0.3 \mathrm{~mm}$, spiral angle $\beta_{1}=8^{\circ}, \quad \delta_{1}=2^{\circ}$.

Parameters of Gear 2: normal module ${ }^{m_{n 1}}=8 \mathrm{~mm}$, normal pressure angle ${ }^{\alpha_{n 1}}=20^{\circ}$, the number of teeth ${ }^{z_{2}}=32, \delta_{2}=2^{\circ}$, gear axis crossing angle $\Sigma=16^{\circ}$.

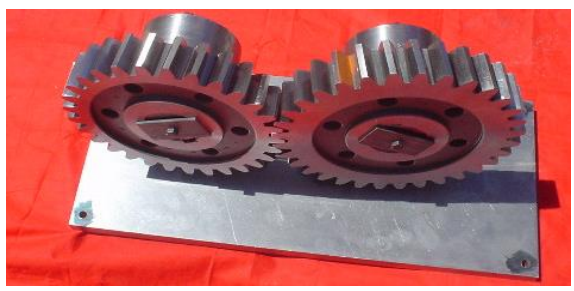

Figure 5. Cross-axis Thickening of Non-involute Gear

\subsection{Computing Model}

A pair of teeth meshing with each other is selected to be modeled according to the spatial position of the gears, and the two gears axes cross with each other at an angle. The contact area of the gears is discreted with meshless method, and the rest is divided into the finite element mesh. The Contact line and the other stress concentration area of the gear are discreted using this meshless method and the rest is discreted with the finite element method. As shown in Figure 6. This numerical model can be established with enough ac- 
curacy and computational efficiency, and such problems can be solved on the PC.

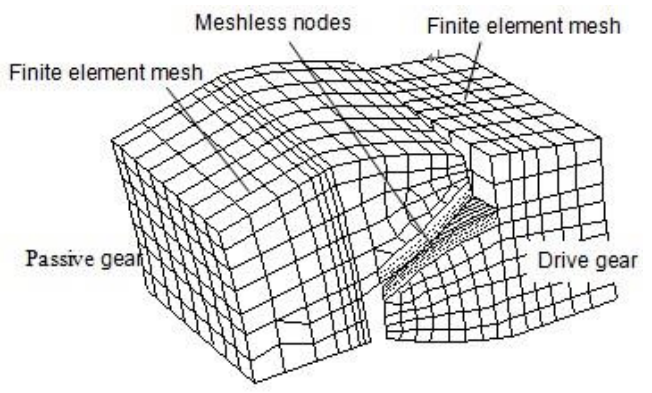

Figure 6. Numerical Analysis Model

Fix the passive gear axes, add torque on the drive gear, and calculate the contact force with the penalty function method.

\subsection{Computing Results}

In order to verify the correctness of the results, the tooth surface contact stress calculated by the finite element method and the classical theory of contact mechanics are compared.

Figure 7a) shows the results of the finite element method, Figure 7b) shows the results of this paper, Figure 7c) shows the stress distribution in the theory of contact mechanics. The principal shear stress contours are given by Figure 7a) and Figure 7b). Compared with Figure 7c) it can be known that the contact stress distribution calculated here is much closer with the classical theory and the rest is little different. The main reason for this result is the stress concentration of the contact area, and stress gradient is large, so it is difficult to calculate the very accurate results by the finite element method. In addition, the contact area is identified by the contact stress in Figure 7a) and Figure 7b) [6], the contact area calculated here is completely line contact, which is consistent with the theoretical design (Theoretical design see conference[5]).
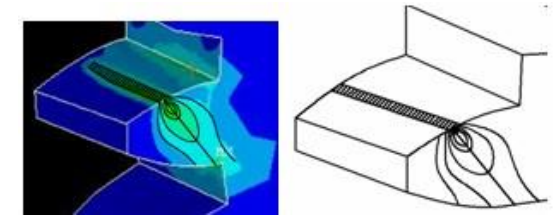

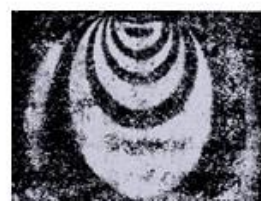
b) Computing Result
c) Theretical Result
a) FEM Computing

Figure 7. Computing Results

By extracting the maximum compressive stress value of the surface, the contact stress with rated load is compared. The result of the finite element method is $926.3 \mathrm{MPa}$, the result calculated in this paper is $819.2 \mathrm{MPa}$. The difference of the results due to the stress concentration of the contact area and the larger stress gradient, and the finite element method results are often too large. 


\section{Failure Analysis of Helical Gear Tooth Root Bending}

\subsection{Computing Background}

A new type of helical gear (as shown in Figure 8) appears broken teeth problem many times during use. Simulation method mentioned in this paper is used to check the strength, and analyze the reasons for gear damage.

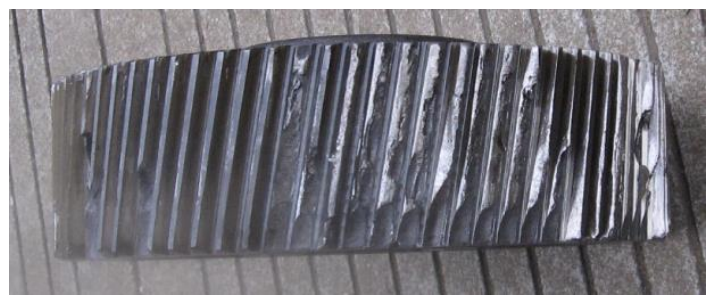

Figure 8. The Destruction of Helical Gear

\subsection{Computing Background}

The mesh is generated automatically using the processing of the finite element software while the no mesh nodes create based on the finite element mesh. And the number of nodes of the tooth root and the crack tip point are increased. In order to simulate the moment at the tooth root, the tangential load is bear at the tooth top.

The gear material is $38 \mathrm{SiMnMo}$, and crack failure criterion is the maximum circumferential stress. The crack propagation step is developed by the computer program, in the paper, 1 / 12 of the tooth root thickness is used.
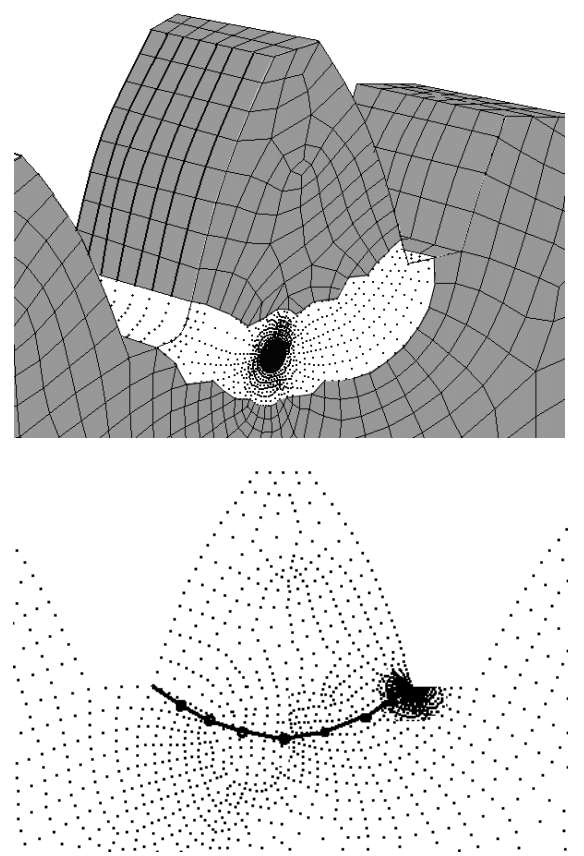

Figure 9. Schematic Diagram of Numerical Model 


\subsection{Computing Background}

Under service load, the maximum tooth root stress is $680 \mathrm{MPa}$, which is below the bending fatigue limit 730MPa. Magnified the load by 1.2, broken lines and crack instability generated at the gear root, which is spread along the gear tooth root to the other side. This agrees with the actual products' failure form. According to this analysis, the damage due to partial load which caused by the gear machining and installation errors, namely contact pressure deviates from the design, focused on the local position of teeth.

\section{Conclusions}

A numerical analysis method used in gear failure analysis is developed, and the method can accurately analyze tooth contact stress and tooth root bending fracture.

The strength and the failure analysis of two types of helical gears are studied with this method, and the application of this method was illustrated. Results of the two examples show that this method is superior to the finite element method In the gear strength analysis function and accuracy. It has a certain practical value in the development and research of the gear.

\section{Acknowledgment}

This work was supported by the Key Program of National Natural Science Foundation of Heilongjiang No.ZD201309, and the Major International Joint Research Program of China (Grant No. 2014DFB70120).

\section{References}

[1] T. Belytschko, Y. Y. Lu and L. Gu, "Element-free Galerkin-methods", Int J numer Meth Engng, vol. 37, (1994), pp. 229-256.

[2] S. N. Atluri, "A new meshless local Petrov-Galerkin (MLPG) approach in computational in mechanids", vol. 22, no. 2, (1998), pp. 117-127.

[3] Babuska, "The Partition of Unity Method", Int. J. for Numer. Mehtods in Enggrg", vol. 40, (1997), pp. 727-758.

[4] F. Guo, W. M. Zhao and G. X. Li, "Polygon Meshless Method: A New Meshless Method Based on MLPG Approach", Journal of Nanjing University of Science and Technology", (2007), pp. 317-322.

[5] G. X. Li, J. M. Wen, F. L. Liu and X. Li, "Profile Errors and Axial Errors of Noninvolute Beveloid Gears with Crossed Axes", Journal of Nanjing University of Science and Technology, vol. 28, no. 6, (2004), pp. 585-589.

[6] X. M. Wu, G. X. Li, W. M. Zhao and F. Guo, "Numerical Analysis Method of Complex Tooth Surface Gear Mesh Contact Region”, Chinese Journal of Mechanical Engineering, vol. 6, no. 43, (2007), pp. 3136. 
International Journal of Control and Automation Vol. 8, No. 1 (2015) 